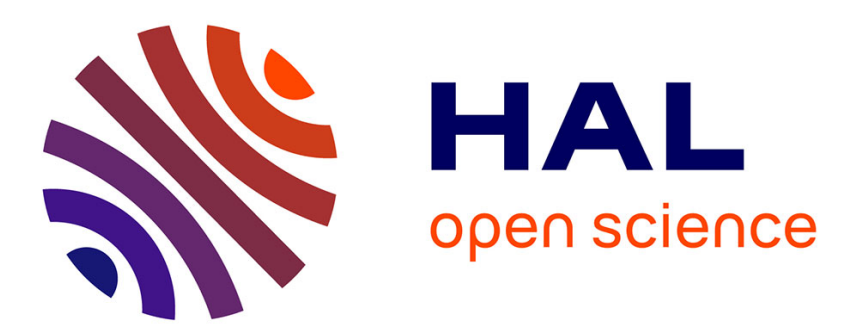

\title{
Aides-soignantes et infirmières, au cœur de l'hôpital
}

Anne-Marie Arborio, Sophie Divay

\section{To cite this version:}

Anne-Marie Arborio, Sophie Divay. Aides-soignantes et infirmières, au cœur de l'hôpital. Sciences humaines, 2019, Les Grands Dossiers, décembre 2018-janvier/février 2019 (53), pp.28-31. hal$02995047 \mathrm{v} 1$

\section{HAL Id: hal-02995047 \\ https://hal.science/hal-02995047v1}

Submitted on 8 Nov 2020 (v1), last revised 1 Feb 2021 (v2)

HAL is a multi-disciplinary open access archive for the deposit and dissemination of scientific research documents, whether they are published or not. The documents may come from teaching and research institutions in France or abroad, or from public or private research centers.
L'archive ouverte pluridisciplinaire HAL, est destinée au dépôt et à la diffusion de documents scientifiques de niveau recherche, publiés ou non, émanant des établissements d'enseignement et de recherche français ou étrangers, des laboratoires publics ou privés. 


\title{
Aides-soignantes et infirmières, au coeur de l'hôpital
}

\author{
Anne-Marie Arborio et Sophie Divay \\ Sciences Humaines, Grands Dossiers n 53 \\ Décembre 2018 / Janvier-février 2019
}

\begin{abstract}
Anne-Marie Arborio, Maître de conférences en sociologie, Laboratoire d'Economie et de sociologie du travail (Aix Marseille Univ, CNRS, LEST, Aix-en-Provence, France)

Sophie Divay, sociologue, maîtresse de conférences, Centre d'étude et de recherche sur les professionnalisations (Université de Reims Champagne Ardenne)
\end{abstract}

Si les médias ont souvent mis les médecins sur le devant de la scène pour illustrer l'héroïsme des soignants, la récente crise sanitaire, due à l'épidémie de Covid-19, a mis en évidence la réalité du soin à l'hôpital où des équipes plurielles sont à l'oeuvre au quotidien : d'une part, les malades, quelles que soient la raison et la durée de leur séjour, ont besoin d'être aidés pour effectuer les actes basiques de la vie quotidienne (toilette, habillage, alimentation, gestion des besoins élémentaires) ; d'autre part, la division du travail à l'hôpital fait que les prescriptions médicales doivent être appliquées par des «petites mains ». Ces tâches sont aujourd'hui assurées par les aides-soignantes et les infirmières, les deux catégories les plus nombreuses de l'ensemble des personnels des établissements de santé. En 2018, la Drees1 compte ainsi 344000 infirmières et 245000 aides-soignantes qui assurent 24 heures sur 24 l'essentiel de la présence soignante dans les établissements de santé, majoritairement comme agents de la fonction publique hospitalière (aux 3/4) ou en CDI dans les établissements privés. Elles sont encore plus nombreuses si l'on y ajoute leurs homologues des Ehpad2 - qui tiennent souvent lieu, en particulier la nuit, de seule présence soignante -, ainsi que celles exerçant à domicile. Et ces effectifs n'ont cessé de croître ces dernières années.

L'emploi du féminin pour nommer ces deux métiers peut surprendre, tant le " masculin neutre » est répandu. C'est que de façon persistante, les hommes sont très minoritaires dans ce domaine professionnel (moins de $10 \%$ ). L'attrait des femmes pour ces métiers est souvent rapporté à leur goût pour le soin et le souci des autres, qui viendraient «naturellement » prolonger le rôle domestique qu'elles jouent auprès de leurs enfants ou de leurs parents malades. À la maison comme au travail, elles mobiliseraient ainsi des « qualités personnelles », telles que la bienveillance ou la compassion. Mais cette interprétation naturalisante masque la construction sociale de la division du travail sexuée en vigueur dans l'espace familial, reproduite dans l'espace professionnel où elle empêche la reconnaissance salariale de ces compétences invisibilisées.

\section{La construction du métier d'infirmière}

Après avoir été un lieu d'hébergement des indigents, soumis aux jeux de pouvoirs religieux et locaux (Gutton, 2002), l'hôpital s'est transformé peu à peu en une institution de soins médicalisés, ouverte à tous les malades. La qualification - en plus de la laïcisation - du personnel placé en contact direct et continu avec les patients est apparue de plus en plus comme une nécessité. Si le terme « infirmier » désignait encore au XIXe siècle tout personnel laïc des hôpitaux, il a pris peu à peu un sens plus restreint pour être réservé aux employés qualifiés, formés dans des écoles, puis dotés d'un diplôme d'État (DE), exerçant dans un cadre réglementé. La fonction d'une infirmière a cependant longtemps été limitée à l'exécution docile des prescriptions du médecin, même si ses tâches se sont complexifiées et diversifiées sous l'effet de l'évolution des techniques médicales et chirurgicales.

1 Direction de la recherche, des études, de l'évaluation et des statistiques, ministère des Solidarités et de la Santé. 
2 Établissement d'hébergement pour personnes âgées dépendantes.

Les revendications d'autonomisation et de valorisation du métier portées par les associations professionnelles ont été fructueuses. La loi de 1978 qui reconnaît un « rôle propre » aux infirmières a fait date : ces dernières sont désormais autonomes et responsables d'un ensemble d'actes répertoriés. La création du corps des infirmières générales en 1975, puis d'un service infirmier en 1991 dans l'organigramme de l'hôpital, les inscrit en outre dans une hiérarchie infirmière indépendante. En 2009, l'« universitarisation » de leur formation a valorisé la profession en attribuant le grade de licence au DE d'infirmier, jusqu'alors maintenu au niveau bac + 2 malgré l'allongement des années d'études qui y menaient; sans oublier l'instauration, en 2018, d'un DE d'infirmière de pratique avancée (IPA) de grade master, et la constitution en 2019 d'une section universitaire en sciences infirmières, ouvrant la voie vers une carrière d'enseignantchercheur.

De création plus récente, le titre d'aide-soignante a été inventé dans l'après-guerre pour reclasser le personnel écarté des soins car non titulaires du DE. Catégorie de reclassement plutôt que métier à proprement parler, le contenu de ses tâches est longtemps resté flou, défini négativement par l'exclusion de tout soin médical et par la dépendance au travail infirmier. Les aides-soignantes exercent, de fait, par délégation du rôle propre infirmier, sans réelle autonomie. La division du travail engendrée dans ce cadre est emblématique du processus que les sociologues analysent à l'aide de la notion de " sale boulot », défini comme l'ensemble des tâches les moins valorisées d'une catégorie de main-d'oeuvre qui sont confiées à une catégorie hiérarchiquement inférieure. La mise en place d'une formation d'aide-soignante qui s'est densifiée au fil des années, débouchant sur un titre professionnel, reconnaît cependant la nécessité de compétences spécifiques au métier. Mais ce titre, fût-il reconnu diplôme d'État depuis 2007, reste un diplôme équivalent à un niveau BEP ou CAP, et laisse les aides-soignantes à distance des infirmières en termes de savoirs comme en termes de pratiques et par suite de prestige social. La révision en cours de leur référentiel de formation, si elle aboutissait à la mise en équivalence du diplôme avec un niveau supérieur, mettrait fin à un défaut de reconnaissance des exigences accrues du métier et d'un recrutement d'élèves dont la majorité est aujourd'hui bachelières.

\section{La frontière floue entre infirmière et aide-soignante}

Le terme d'aide-soignante pourrait laisser penser à une collaboration directe avec l'infirmière, mais le fameux « binôme » infirmière-aide-soignante n'a pas toujours cours dans le quotidien des services. Une organisation strictement séparée du travail est parfois préférée, notamment dans les services très techniques (en réanimation, en salle de réveil, en dialyse, aux urgences, au bloc, etc.). Ailleurs, comme en soins de suite et de réadaptation ou encore en Ehpad, où les effectifs de soignantes ne sont pas réglementés et où le manque de personnel est récurrent, les aidessoignantes sont souvent contraintes d'assumer des tâches réservées aux infirmières en plus de leurs propres tâches. Ces «glissements de tâches » illégaux ne sont pas sans les mettre dans l'embarras : non seulement elles effectuent les toilettes (parfois jusqu'à une vingtaine en une matinée) et les « changes » des personnes incontinentes (plusieurs «tournées » par jour et par nuit), mais il leur arrive aussi de distribuer des médicaments, de faire des soins infirmiers (aspiration, changement de «canule de trachéo », injections intramusculaires, « surveillance de perf $\gg \ldots)$.

La position des aides-soignantes paraît ainsi subordonnée dans la division du travail mais avec des variations selon les services. Si elles relèvent parfois des signes de mépris de la part de malades ou de collègues qui les blessent, elles sont aussi souvent valorisées par leur proximité aux malades et la connaissance intime qui en résulte. Cette rhétorique construite autour d'une relation privilégiée aux malades leur permet de se valoriser, en creux, par différenciation d'avec les infirmières qui leur apparaissent comme surchargées de tâches administratives ("paperasserie », dossiers informatisés à remplir...) et davantage soumises à la pression gestionnaire. Les aides- 
soignantes ne sont cependant pas à l'abri des pressions temporelles qui s'exercent dans les établissements de santé : elles en rendent compte au travers de l'indicateur du temps passé auprès de chaque malade qui leur paraît sans cesse diminuer.

\section{Des conditions matérielles difficiles}

Les conditions d'exercice se sont en effet durcies partout, pour les aides-soignantes comme pour les infirmières, sous l'effet de diverses évolutions. Aux restrictions budgétaires s'ajoutent un alourdissement de la charge physique et émotionnelle de travail, une inflation de normes à suivre sous forme de protocoles, règles, tableaux de bord, sans compter la multiplication des contrôles et évaluations récurrentes des pratiques (certification, « patient traceur», etc.)

Par ailleurs, comme d'autres métiers de la santé, en particulier pour ceux qui comme ici sont très majoritairement exercés par des femmes, l'articulation des horaires continus de travail avec la vie de famille n'est pas toujours aisée. Et si l'ouverture de la possibilité du travail en postes de 12 heures, à des fins de rationalisation budgétaire, apparaît de façon paradoxale, comme une avancée vers le «temps choisi » (Vincent, 2014), les soignantes y gagnent en jours de congé et réduisent leurs trajets travail-domicile (parfois très longs en Île-de-France), mais elles y perdent en confort de travail avec un surcroît de fatigue et de tension nerveuse.

De telles conditions de travail ne sont pas sans conséquences sur la santé des agents. Les établissements de santé sont l'un des secteurs où les salariés ont le plus recours aux arrêts maladie, en lien direct avec leurs conditions de travail3. Pour assurer le remplacement des absences, les cadres de santé « jonglent » avec la mobilisation de précaires, l'exercice de pressions sur les personnels en poste, le fonctionnement dit « en mode effectif dégradé » avec l'autorisation de leur hiérarchie. Le cercle vicieux d'un tel " management » de la pénurie guette alors les équipes, les remèdes à l'absentéisme entraînant stress, conflits, épuisements, burn-out, et donc... davantage d'arrêts pour maladie.

Le malaise - voire le mal-être - de ces soignantes trouve sa source dans un faisceau de causes interdépendantes, symboliques et matérielles. Le thème de la non-reconnaissance est très répandu. Dans certains services, les infirmières se sentent méprisées par des médecins ou chirurgiens, autoritaires et irrespectueux4; quant aux aides-soignantes, elles disent être «invisibles » ou ignorées par ces mêmes médecins. Le niveau de leurs salaires traduit aussi la faible valeur sociale accordée à ces différents professionnels : ainsi, dans les établissements de santé, les salaires moyens des aides-soignantes et même des infirmières sont-ils largement inférieurs à ceux des médecins. La présence remarquée d'aides-soignantes parmi les gilets jaunes au cours de l'hiver 2018-2019 a mis au jour les difficultés matérielles de certaines d'entre elles, en particulier lorsqu'elles ont seules la charge d'enfants. 
3 C. Pollak et L. Ricroch, « Arrêts maladie dans le secteur hospitalier : les conditions de travail expliquent les écarts entre professions », Drees, Études et résultats, n $^{\circ}$ 1038, 2017.

Il y a plus grave et plus pénible encore pour l'ensemble des soignantes. La pression (temporelle, gestionnaire) impose de travailler dans la précipitation, souvent de façon décousue, et les met ainsi dans l'obligation de «mal faire leur travail », de se faire mal et de faire mal pour parvenir à faire tout ce qu'il y a à faire en trop peu de temps et avec pas assez de moyens. Et l'ombre de la « maltraitance » plane. « Faire manger » une patiente âgée et grabataire en dix minutes, au risque de lui faire faire une fausse route. Ou encore, faire une toilette à un patient tétraplégique en un quart d'heure, là où il faudrait pouvoir prendre le temps d'établir une relation par le toucher, en pratiquant des massages... Qu'on ne s'y trompe donc pas, ces situations ne sont pas dues à de la malveillance de la part des personnels, mais à de la maltraitance organisationnelle qui laisse les soignantes seules face à elles-mêmes et aux patients. Le travail collectif est empêché : les moments de transmission entre équipes diminués, voire supprimés ; les soins et les changes ne peuvent plus se faire à deux, etc.

Les mauvaises conditions de travail des soignants ont été dénoncées à travers différents mouvements de protestation, d'abord dans les services d'urgences des hôpitaux, puis à la suite de l'épidémie de Covid-19. Toutefois, le "Ségur de la santé »n'a pas débouché sur de réelles solutions de fond, dans le sens où les problèmes structurels de l'hôpital public n'ont pas véritablement été traités.

\section{Quelles perspectives de carrière ?}

Dans le contexte socio-économique actuel, on peut s'interroger sur le devenir de métiers qui, bien qu'indispensables, manquent d'attractivité. La pénibilité du travail est-elle au moins compensée par les carrières offertes à ces soignantes ?

Les associations d'aides-soignantes plaident depuis plusieurs années en vain pour davantage d'autonomie, pour un rôle propre d'aide-soignante qui autoriserait l'installation en libéral. Faute de promotion collective, les aides-soignantes ne peuvent envisager que de petits déplacements individuels dans l'espace social : certaines jeunes aides-soignantes pourraient plus facilement que leurs aînées, moins dotées en titres scolaires, envisager de devenir infirmières mais, on l'a dit, elles ne le souhaitent pas toujours, et les carrières horizontales, à la recherche d'un meilleur établissement ou d'un meilleur poste, sont les seules voies pour celles qui restent. Elles imposent parfois de passer un autre diplôme de même niveau, par exemple celui d'auxiliaire de puériculture pour s'assurer de travailler en pédiatrie ou en crèche. La fonction d' " assistant médical », introduite en 2019, pourrait être ouverte aux aides-soignantes après un complément de formation. Elle ajoute une voie de promotion, toujours horizontale, avec des conditions de travail - en cabinet médical, à proximité du médecin - assez différentes des autres. 
4 À ce propos, voir le documentaire de J. Le Maire Burning out. Dans le ventre de l'hôpital (2016). 
Les infirmières regrettent aussi un manque de perspective professionnelle, même si elles ont davantage de possibilités de carrière en se spécialisant pour devenir infirmières anesthésistes, infirmières de bloc, puéricultrices, ou en devenant cadres de santé. Mais la spécialisation peut aussi se révéler enfermante car on ne sort pas facilement du bloc ou de la pédiatrie et la fonction de cadre de santé exige de "faire le deuil de son métier », passer de la clinique à la gestion et au management, un pas que nombre de soignantes ne sont pas prêtes à franchir. Reste l'exercice en libéral. Si l'infirmière gagne en liberté, elle doit se convertir en chef d'entreprise et « faire du chiffre », et donc ne pas compter ses heures ni les kilomètres parcourus.

Pour la profession infirmière, mieux organisée, de nouveaux horizons s'ouvrent depuis peu, les IPA ayant l'opportunité de se spécialiser dans les soins, d'obtenir un grade de master et d'élargir leur champ de compétences cliniques, mais sous un fort contrôle médical, tous les masters créés jusqu'à présent étant rattachés à des facultés de médecine. Il reste à suivre l'accueil que leur réserveront les autres infirmières généralistes et les médecins, ainsi que la mise en place concrète de leurs fonctions, avec les éventuels transferts de charges d'une catégorie à l'autre...

\section{Pour aller plus loin}

F. Acker, A.-M. Arborio, «Infirmière et aide-soignante », in D. Lecourt (Dir.), Dictionnaire de la pensée médicale, PUF, 2004.

A.-M. Arborio, Un personnel invisible. Les aides-soignantes à l'hôpital, 2e éd., Economica, 2012.

S. Divay, Soignantes dans un hôpital local. Des gens de métier confrontés à la rationalisation et à la précarisation, EHESP, 2013.

S. Divay, «Les carrières des infirmières, plus horizontales que verticales », Net.Doc, $\mathrm{n}^{\circ} 90$, mars 2012.

I. Feroni et A. Kober, «L'autonomie des infirmières. Une comparaison France/Grande-Bretagne $»$ Sciences sociales et santé, vol. XIII, $\mathrm{n}^{\circ} 3$.

J.-P. Gutton, (dir.), Les administrateurs d'hôpitaux dans l'Europe moderne, P.U.L., 2002.

F. Vincent, "Travailler pour son "temps de repos" ? Sens et usages du temps hors travail des soignantes en 12 heures à l'hôpital public ", Temporalités. Revue de sciences sociales et humaines, 2014, n²0. [En ligne : http://temporalites.revues.org/2896] 